\title{
ARTICLE OPEN Regulatory feedback response mechanisms to phosphate starvation in rice
}

\author{
Ishan Ajmera $\mathbb{D}^{1,2}$, Jing Shi ${ }^{3,4}$, Jitender Giri ${ }^{5}$, Ping Wu ${ }^{3}$, Dov J. Stekel $\mathbb{D}^{1}$, Chungui Lu ${ }^{1,6}$ and T. Charlie Hodgman (D) ${ }^{1,2}$
}

Phosphorus is a growth-limiting nutrient for plants. The growing scarcity of phosphate stocks threatens global food security. Phosphate-uptake regulation is so complex and incompletely known that attempts to improve phosphorus use efficiency have had extremely limited success. This study improves our understanding of the molecular mechanisms underlying phosphate uptake by investigating the transcriptional dynamics of two regulators: the Ubiquitin ligase PHO2 and the long non-coding RNA IPS1. Temporal measurements of RNA levels have been integrated into mechanistic mathematical models using advanced statistical techniques. Models based solely on current knowledge could not adequately explain the temporal expression profiles. Further modeling and bioinformatics analysis have led to the prediction of three regulatory features: the $\mathrm{PHO} 2$ protein mediates the degradation of its own transcriptional activator to maintain constant PHO2 mRNA levels; the binding affinity of the transcriptional activator of PHO2 is impaired by a phosphate-sensitive transcriptional repressor/inhibitor; and the extremely high levels of IPS1 and its rapid disappearance upon Pi re-supply are best explained by Pi-sensitive RNA protection. This work offers both new opportunities for plant phosphate research that will be essential for informing the development of phosphate efficient crop varieties, and a foundation for the development of models integrating phosphate with other stress responses.

npj Systems Biology and Applications (2018)4:4; doi:10.1038/s41540-017-0041-0

\section{INTRODUCTION}

Ensuring a secure and sustainable food supply for the growing human population is a global priority. Current agricultural production is unsustainable because of the increasing scarcity of fresh water, limited availability of land, global climate change, degradation of soil and the depletion of fertilizer stocks. Phosphate availability is often the limiting factor in crop growth, and its interactions with other nutrients are complex. ${ }^{1-3}$ The use of phosphate fertilizer is both economically and ecologically unsustainable, because phosphate stocks are a non-renewable mineral resource, ${ }^{4}$ and the run-off of surplus fertilizer damages the environment. ${ }^{5}$ An alternative to the high use of phosphate fertilizer is to develop crops with increased phosphorus use efficiency, ${ }^{6,7}$ though success on this front has been hampered by the complexity of phosphate chemistry and plant responses to the low availability of soil phosphate. ${ }^{8}$ It is essential to better understand the mechanisms involved in regulating phosphate uptake and homeostasis in plants.

Inorganic phosphate $(\mathrm{Pi})$ starvation triggers a broad range of adaptive responses at the biochemical, genetic, physiological, morphological, anatomical and rhizospheric scales. These include derepression of high-affinity phosphate transporters on the outer cell membranes, a metabolic shift to use of sulfate, reduced photosynthesis, reduced shoot growth, altered root architecture (including lateral root formation and long root hairs), formation of aerenchyma, and secretion of exudates into the surrounding soil to solubilize phosphate, stimulate soil bacteria and attract filamentous fungi to receive $\mathrm{Pi}$ from long distances. Pi-starvation responses (PSRs) are themselves complex, but regulated by mechanisms largely conserved between Arabidopsis and rice. ${ }^{9}$

The key transcriptional activator (TA) in Pi starvation signaling is phosphate starvation response 1 in Arabidopsis, and its ortholog OsPHR2 in rice (Oryza sativa), see Supplementary Fig. 1. The gene for the latter is constitutively expressed, but the protein does not become active until it is both free to migrate to the nucleus (which occurs with the proteolysis of SPX4 under low cytosolic Pi conditions), ${ }^{10}$ and be sumoylated (which is a general feature of plant abiotic stress). ${ }^{11}$ The active form results in the expression of numerous Pi-starvation-induced genes, including some to make better use of the current cellular phosphate and a microRNA, miR399, which is the systemic integrator defining $\mathrm{Pi}$ demand across the whole plant. Mostly miR399 is expressed in shoots and translocated to the root via the phloem, causing degradation of $\mathrm{PHO} 2$ mRNA through target mimicry. The $\mathrm{PHO} 2$ gene encodes a ubiquitin-conjugating E2 enzyme (UBC24) that indirectly inhibits high-affinity Pi transporters (PHTs) and phosphate 1 (PHO1), the transporter which puts $\mathrm{Pi}$ into the xylem for systemic distribution. $^{12-16}$ The PHO2 mRNA-miRNA399 interaction is also modulated by a long non-coding RNA, At4 in Arabidopsis and induced by phosphate starvation 1 (IPS1) in rice. ${ }^{17,18}$ OsIPS1 is induced by $\mathrm{Pi}$ starvation and has a region partially complementary to miR399, ${ }^{19}$ which enables it to sequester miR399, reducing the availability of free miR399 for degradation and inhibiting complete silencing of $\mathrm{PHO} 2$ mRNA.

\footnotetext{
${ }^{1}$ School of Biosciences, The University of Nottingham, Sutton Bonington Campus, Sutton Bonington, Loughborough LE12 5RD, UK; ${ }^{2}$ Centre for Plant Integrative Biology, University of Nottingham, Sutton Bonington, Loughborough LE12 5RD, UK; ${ }^{3}$ State Key Laboratory of Plant Physiology and Biochemistry, College of Life Science, Zhejiang

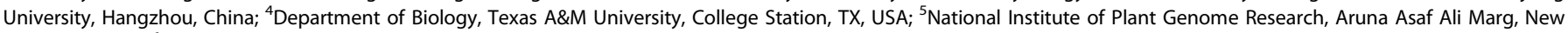
Delhi, India and ${ }^{6}$ School of Animal, Rural and Environmental Sciences, Nottingham Trent University, Nottingham NG1 4FQ, UK

Correspondence: Chungui Lu (chungui.lu@ntu.ac.uk) or T. Charlie Hodgman (charlie.hodgman@nottingham.ac.uk)

Ping $\mathrm{Wu}$ is deceased
}

Received: 1 March 2017 Revised: 14 November 2017 Accepted: 24 November 2017

Published online: 8 January 2018 

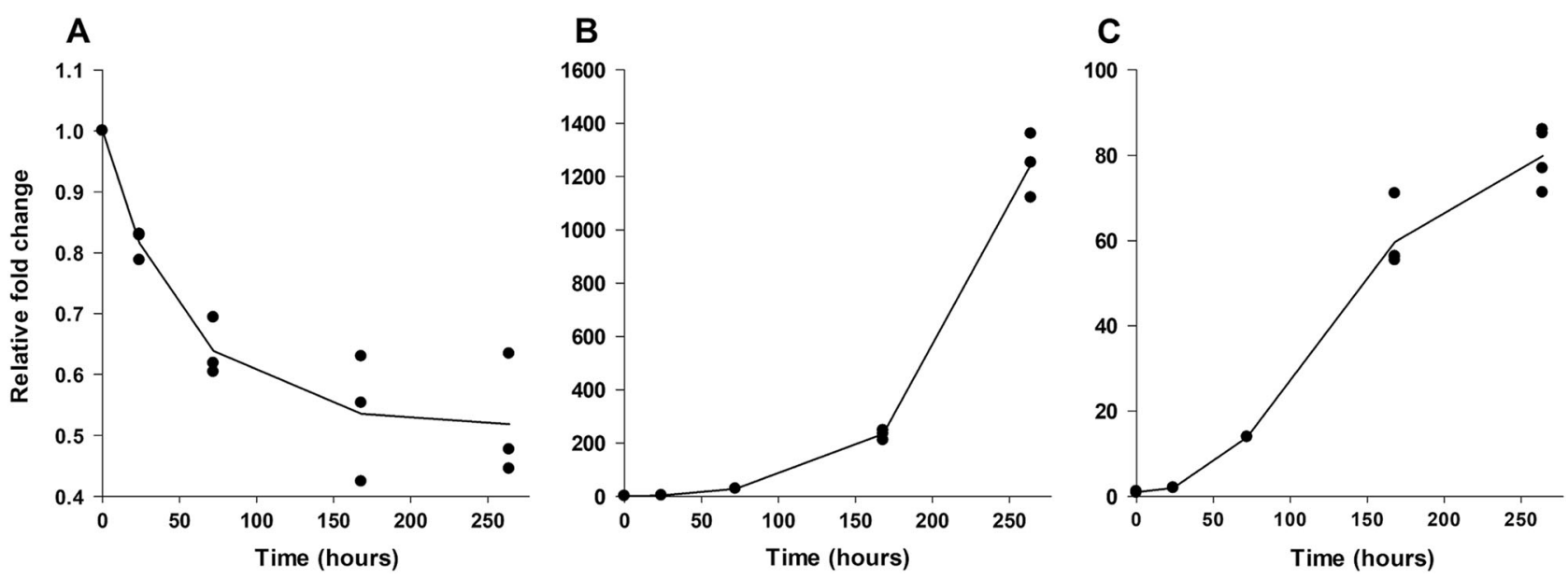

Fig. 1 Expression profiles of a PHO2, b IPS1 and c miR399 in Pi-starved rice seedling (cv. Nippobare) roots. The expression levels are relative to the $+\mathrm{P}$ condition at time zero. Relative expression levels were normalized to that of the internal control, Os-ACTIN

This work integrates mathematical modeling, informatics and laboratory techniques to analyze the molecular regulation of phosphate acquisition during deficiency, with particular emphasis on $\mathrm{PHO} 2$ and IPS1 transcript dynamics. Three new regulatory features are predicted: first, that factors other than miR399 are responsible for the early decline in $\mathrm{PHO} 2$ transcript levels; second, that $\mathrm{PHO} 2$ appears to negatively regulate its own expression; and third, that the high levels of IPS1 and its rapid drop upon Pi resupply are best explained by Pi-sensitive RNA protection (RP). The resulting mathematical model also provides a base for studying other aspects of the phosphate-starvation response, including its combined effect with other stresses.

\section{RESULTS}

Delayed expression of miR399 compared with PHO2 mRNA loss A time-course expression data for miR399, alongside data for IPS1 and $\mathrm{PHO} 2$ transcripts to test reproducibility against previously published data, were obtained for plants under Pi stress, using quantitative RT-PCR. The level of PHO2 mRNA shows a progressive drop, most steeply at earliest times, to about half the original level over 11 days of Pi stress (Fig. 1a); IPS1 appears to increase exponentially to extremely high levels over the same timescale (Fig. 1b). In contrast, there is a delay of at least $24 \mathrm{~h}$ before miR399 levels begin to increase in a roughly sigmoid manner (Fig. 1c). From these data, it seems unlikely that early decline in $\mathrm{PHO} 2$ mRNA levels can be directly attributed to the effects of miR399.

A new mathematical model of the molecular regulation of phosphate uptake

The published ${ }^{20,21}$ and the presented (Fig. 1) experimental data pose two questions about the regulation of the PHO2 and IPS1. First, what causes the drop in $\mathrm{PHO} 2$ mRNA at early times upon $\mathrm{Pi}$ stress, given that corresponding levels of miR399 are quite low? Second, what causes the extreme elevation of IPS1 in response to $\mathrm{Pi}$ starvation, and its steep and sudden decline following Pi resupply? To address these questions, a new mathematical model for the regulation of phosphate uptake in plants has been developed by adopting the molecular network presented as Fig. $2 a$. The model includes key regulators, both from upstream and downstream of miR399, PHO2 and IPS1: SIZ1, PHR2, PHTs and $\mathrm{PHO} 1$. This is primarily to explore the impact of miR399, $\mathrm{PHO} 2$ and IPS1 on phosphate uptake and allow the model to integrate other aspects of $\mathrm{Pi}$ homeostasis and other stress conditions. ${ }^{22-24}$

This base model, called the Pi Original Model (PiOM), is a system of ten coupled non-linear ordinary differential equations and defined in Supplementary Methods (Equation 1-10). The values of some parameters were identified from the literature, rationally assumed or calculated (see Supplementary Table 2), while some were inferred by fitting the model to the experimental data as described in the Supplementary Table 3. The available experimental data points include: fold changes in the miR399, $\mathrm{PHO} 2$ mRNA and IPS1 levels in response to Pi starvation presented in Fig. 2; and published mRNA-SEQ data, particularly for PHO2. ${ }^{20,21}$ Later, the base model was modified to test five competing hypotheses to explain unknown mechanisms regulating $\mathrm{PHO} 2$ transcription and two potential hypotheses for IPS1 dynamics (see below).

PiOM fits IPS1 and miR399 data but poorly predicts $\mathrm{PHO} 2$ dynamics

The PiOM fit to the experimental (fold change) data for IPS1 and miR399 is good (Fig. 3b, c), including the induction of miR399 and IPS1 and their minimal steady state expression under both Pideficient and sufficient conditions. The level of PHO2 mRNA starts to decline as early as $6 \mathrm{~h}$ following Pi stress but does not disappear completely. ${ }^{20}$ Figure $3 a$ shows that the model reflects the latter, presumably because IPS1 reversibly sequesters the bulk of the miR399 available in the roots. However, the model does not correspond with the early drop in PHO2 mRNA level. This is because the level of miR399 increases in roots after $24 \mathrm{~h}$ of $\mathrm{Pi}$ stress and indicates that something else is affecting $\mathrm{PHO} 2$ transcript levels, particularly at early times.

Potential hypotheses and mechanistic models for early $\mathrm{PHO} 2$ loss Five hypotheses are considered as potential mechanistic explanations for the observed $\mathrm{PHO} 2$ transcript dynamics. These are diagrammatically presented in Fig. $2 b-f$ and mathematically encoded as modified versions of the PiOM base model given in Supplementary Methods (Equations 11-17). The first four hypotheses assume some unknown regulator $\mathrm{Z}$ acting as (i) a Pidependent TA of PHO2 (PdTA), (ii) a Pi-dependent protector of $\mathrm{PHO} 2$ mRNA (PdRP), (iii) a Pi-sensitive binder of PHO2 mRNA causing mutual degradation (PsMD), or (iv) a Pi-sensitive transcriptional repressor of $\mathrm{PHO} 2$ (PsTR). The fifth hypothesis assumes a Pi-sensitive RNase promoting degradation of $\mathrm{PHO} 2$ mRNA in the absence of CytoPi (PsRA). Each hypothesis was tested as an individual model with an attempt to fit the complete data set while using the previously estimated parameters from the PiOM base model as initial values. 


\section{A PiOM}

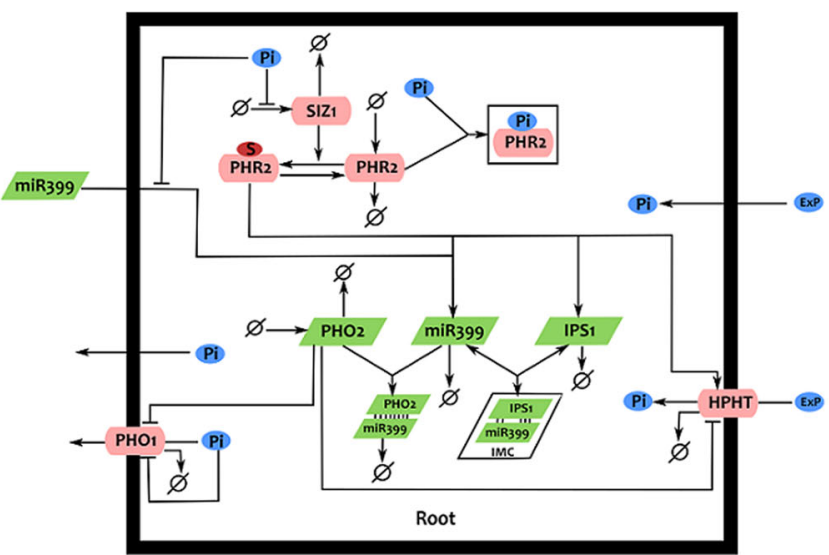

C

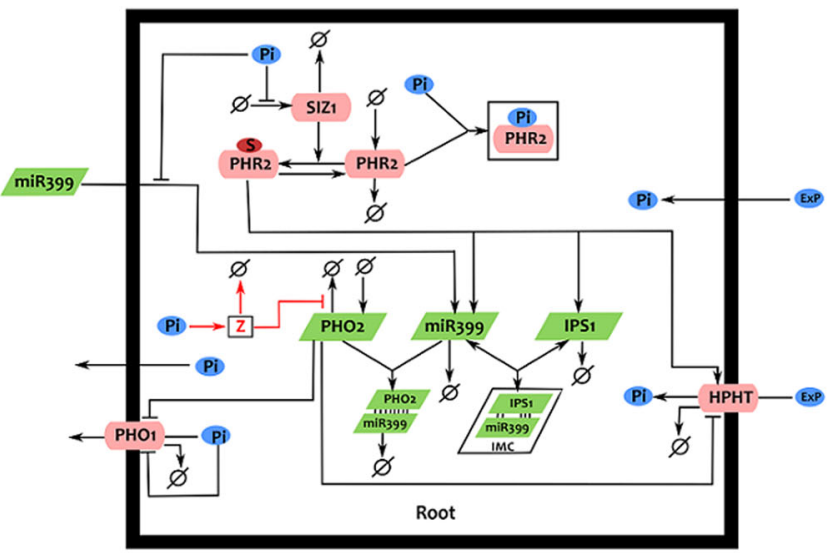

E

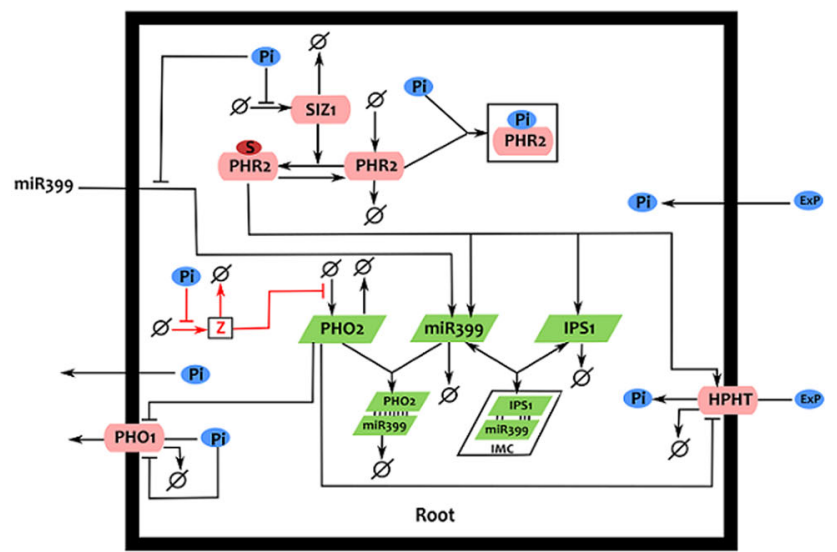

\section{B PdTA}

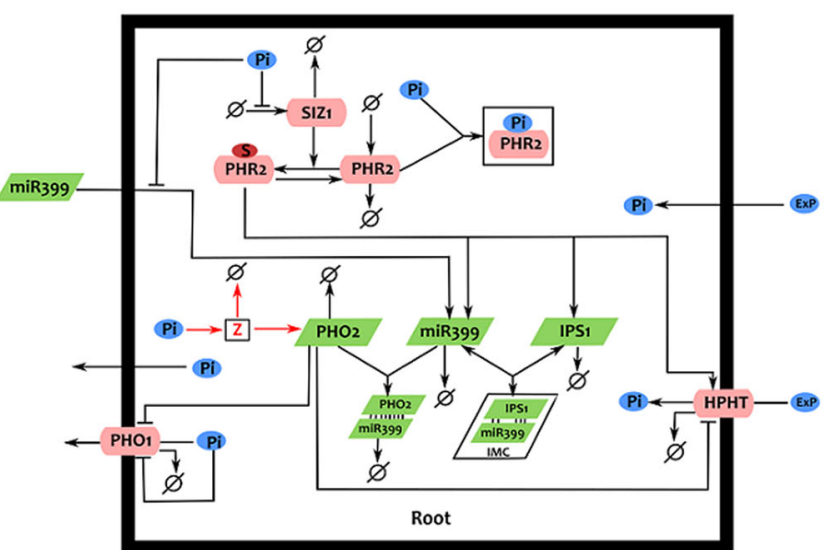

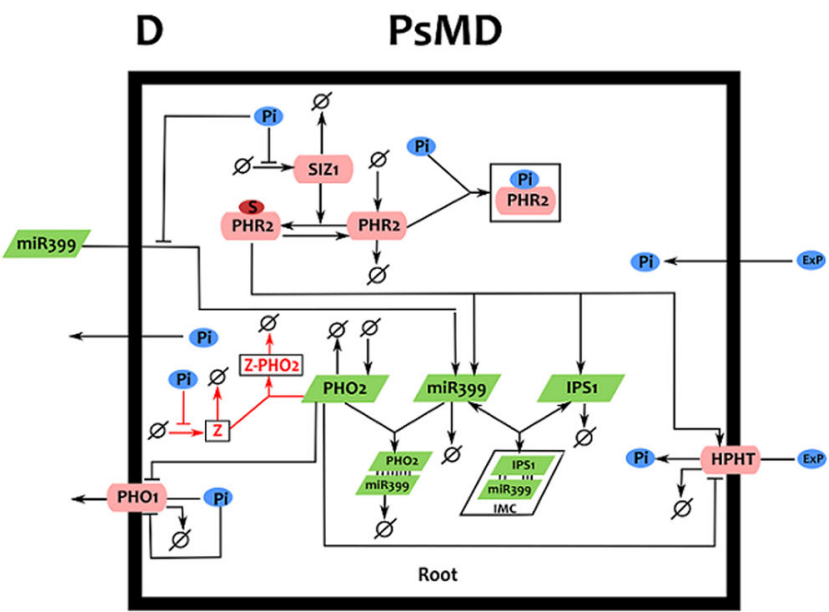

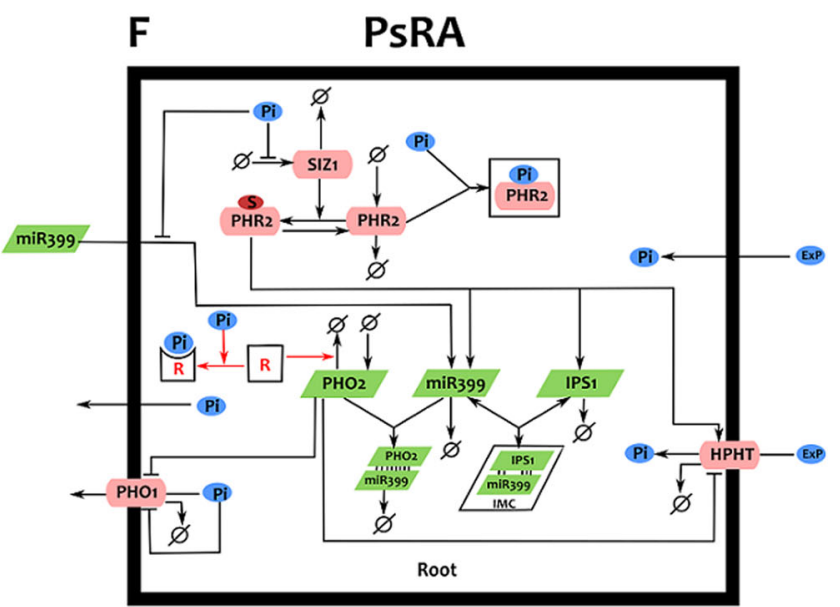

Fig. 2 Schematic representation of the molecular regulation of Pi uptake. a A simplified depiction of the molecular network regulating $\mathrm{Pi}$ acquisition. b-f five hypotheses to explain the observed PHO2 transcript dynamics under Pi-deficient conditions. Panels b-f depict individual hypotheses (models) in a single root cell (thick black box). Red lines and letters denote the assumed reactions and species, respectively. Each hypothesis assumed the presence of an unknown regulator $\mathbf{Z} \mathbf{b}-\mathbf{e}$ or $\mathrm{R}(\mathrm{F})$ acting in different manners. Protein, ligand, mRNA, complexes and $\mathrm{Pi}$ ions are denoted by rectangles (pink), red ellipse, parallelogram (green), squares (transparent) and blue ellipse, respectively. Pointed solid arrows denote direct interactions and fluxes, barred arrows denote inhibition or repression, and dashed lines represent indirect interactions. $\varnothing$ denotes endogenous production and degradation of the molecules

PsTR model best explains the early regulation of $\mathrm{PHO} 2$ in $\mathrm{Pi}-$ deficient condition

All five models show a good fit to the PHO2 (Fig. 4), IPS1 and miR399 data set in Pi-deficient conditions (see Supplementary Figs. 2 and 3) with -2 log-likelihood values lower than the original model. Using the inferred Akaike Information Criterion (AIC), the values from statistical analysis indicate that the hypothesis models offer significantly better fits to the data than the original model, see Table 1. Among the five hypotheses, PdRP gives the lowest AIC value and, statistically, it is the best hypothesis for $\mathrm{PHO} 2$ 

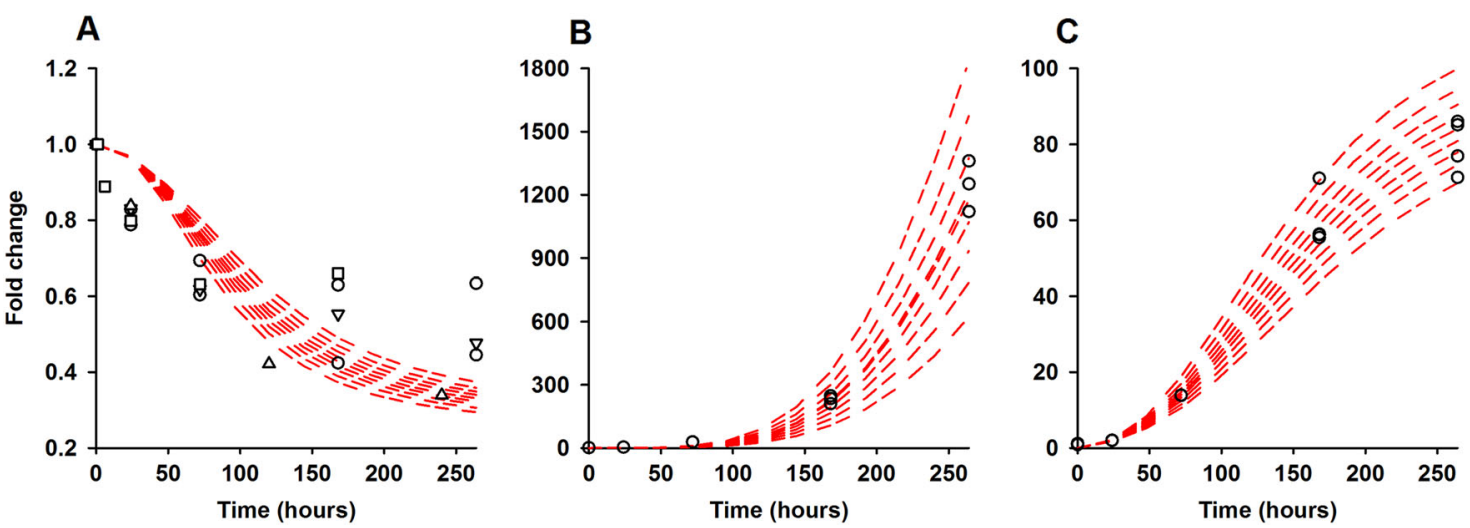

Fig. 3 Model fitting to time-course data. Circle represents the qRT-PCR data. Square and triangles denotes fold change mRNA-seq data adopted from, ${ }^{20,21}$ respectively for a PHO2, b IPS1 and c miR399. Prediction intervals of $80,85,90,95$ and $100 \%$ are shown as red dashed lines. Only three data points for PHO2 fall within the certainty limits of prediction interval of the model, unlike IPS1 and miR399 which almost all lie within the $80 \%$ interval
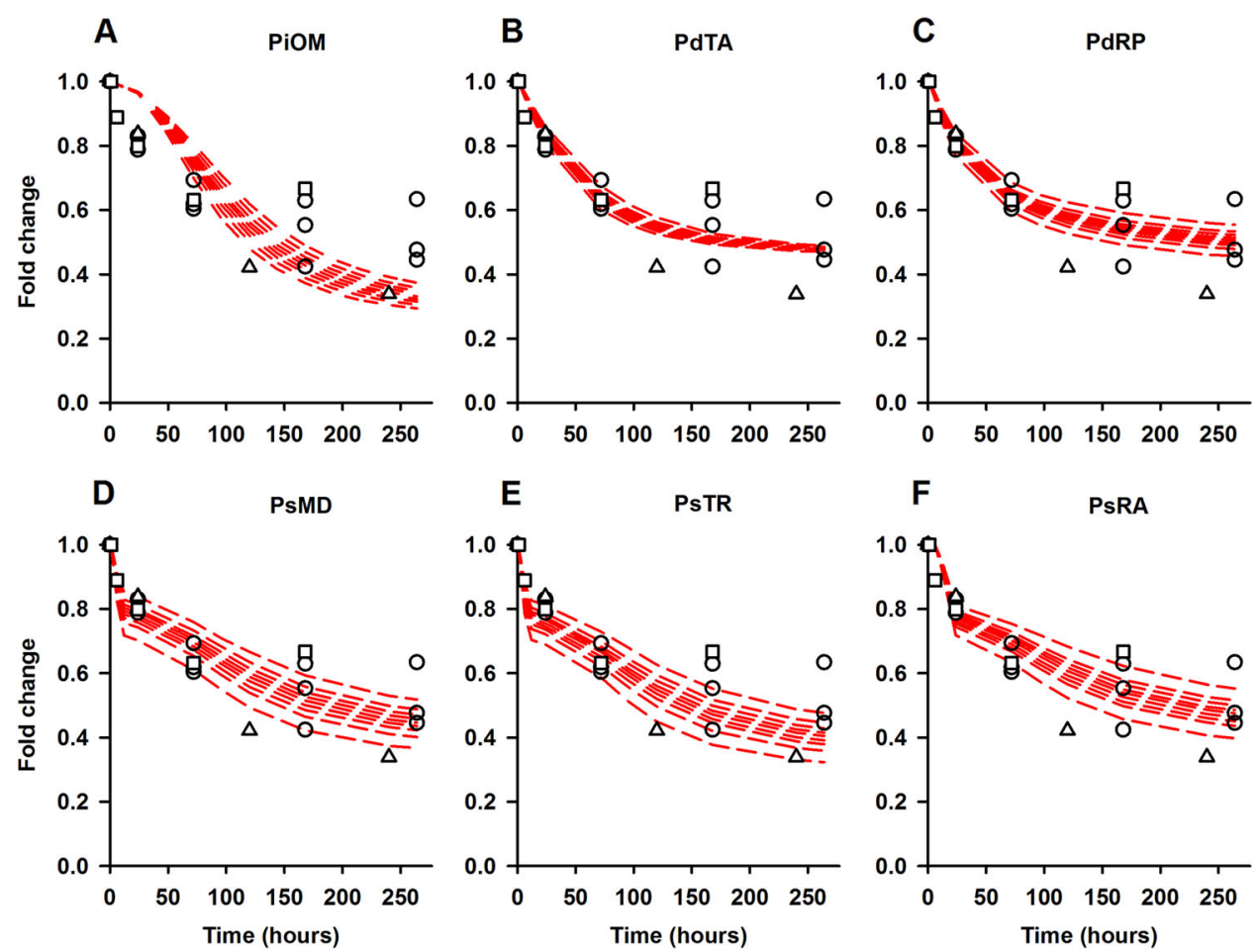

Fig. 4 Fitting of different hypothesis models to PHO2 data. Panel a represents the fits of PiOM model. Panels b-f depicts the fit offered by individual hypothesis, labeled in the respective panel. Red dashed lines represent the $80 \%$ prediction interval of the respective hypothesis model. These simulations have been carried out by sampling parameter values from normal (Gaussian) distributions with means and standard deviations given from the parameter fitting in Monolix. Circle represents the qRT-PCR data. Square and triangles denotes fold change mRNAseq data adopted from, ${ }^{20}, 21$ respectively

transcript dynamics. However, there is a qualitative difference between the models in predicting the drop in $\mathrm{PHO}$ level at 6-h time point following $\mathrm{Pi}$ starvation. This feature of $\mathrm{PHO} 2$ is only captured by PsMD and PSTR models. Altogether, this exhibits a need to distinguish between qualitatively correct and statistically sound models, which can be achieved by experimentally determining when $\mathrm{PHO} 2$ mRNA levels start to decrease upon $\mathrm{Pi}$ stress.

PHO2 mRNA levels from rice roots after $0,3,6$ and $12 \mathrm{~h}$ of $\mathrm{Pi}$ starvation were measured using qRT-PCR (Supplementary Fig. 4). The Pi-sufficient conditions showed no evidence of being regulated by the circadian clock and, if anything, increased slightly over $12 \mathrm{~h}$. In contrast, almost $80 \%$ of PHO2 mRNA was lost by $3 \mathrm{~h}$ of Pi stress but recovered slightly at 6 and $12 \mathrm{~h}$. This result again favors the PsMD and PsTR models. It is difficult to envisage biological examples of PsMD. Biochemically, a PsMD would require a Pi-binding RNA or Pi-binding proteins that are mutually degraded with a target RNA. The first of these is highly unlikely due to mutual electrostatic repulsion, while no evidence has been found for the latter. However, PsTR is more biologically credible as proteins could contain allosteric Pi-binding pockets, making this the most probable model to explain the early $\mathrm{PHO} 2$ transcript 
dynamics. Certainly, further experimental validation is needed to confirm this. The early-time qPCR profile also implies the presence of a feedback mechanism that attempts to maintain $\mathrm{PHO} 2$ transcript levels. For example, $\mathrm{PHO} 2$ might ubiquitinate and hence degrade its own activator, analogous to the maintenance of plant hormone-associated TF levels. ${ }^{25}$ Such a mechanism could also explain why $\mathrm{PHO} 2$ mRNA does not completely disappear.

Table 1. List of AIC, -2 log-likelihood and $P$-value for the hypothesis models

\begin{tabular}{lllllll}
\hline Model & AIC $^{\mathrm{a}}$ & $-2 \mathrm{LL}^{\mathrm{a}}$ & $\mathrm{T}^{\mathrm{b}}$ & $\triangle \mathrm{AIC}^{\mathrm{c}}$ & $\mathrm{k}^{\mathrm{d}}$ & $P$-value \\
\hline PiOM & 238.7 & 142.7 & 48 & - & - & - \\
PdRP & 192.2 & 88.2 & 52 & 46.5 & 4 & $4.14 \mathrm{E}-11$ \\
PdTA & 206.58 & 106.58 & 50 & 32.12 & 2 & $1.43 \mathrm{E}-08$ \\
PsRA & 211.51 & 111.5 & 50 & 27.19 & 2 & $1.69 \mathrm{E}-07$ \\
PsMD & 218.86 & 106.86 & 56 & 19.84 & 8 & $1.88 \mathrm{E}-05$ \\
PsTR & 234.31 & 122.31 & 56 & 4.39 & 8 & $8.96 \mathrm{E}-03$
\end{tabular}

a The AIC and log-likelihood (-2LL) values were generated for each model while estimating parameters using MONOLIX

${ }^{\mathrm{b}}$ total number of parameters from both structural and statistical models

${ }^{c}$ AIC - AIC (of PiOM)

d $\mathrm{T}$ - T(of PiOM)

e Probability $\left(X^{2} \mathrm{k}>\Delta \mathrm{AIC}+2 \mathrm{k}\right){ }^{42} P$-values were calculated using the $X^{2}$ distribution (CHIDIST) function in Microsoft Excel
Anomalies in the predicted profiles of $\mathrm{PHO} 2$ and IPS1 upon Pi resupply

PHO2 and IPS1 transcript levels are observed to undergo a sudden drop within $24 \mathrm{~h}$ following Pi re-supply, ${ }^{20}$ see Fig. 5 . A sudden loss of IPS1 would release the bound miR399, causing the observed rapid loss of PHO2 mRNA. Both the PiOM and PsTR models incorrectly predict the observed drastic drop in mRNA levels of PHO2 and IPS1 (Fig. 5a, c) and the plausible rise in miR399 level in response to Pi repletion (Supplementary Fig. 5a, b). This suggests some extra level of regulation concerning $\mathrm{PHO} 2$ and IPS1, not represented in the current models. The fold change of $\mathrm{PHO} 2$ between $200-500 \mathrm{~h}$ in RNAseq data is larger than those predicted by the model, Fig. $5 a$, $b$.

Potential hypotheses for elevation of IPS1

The IPS1 level is observed to rise more than 1000 fold within 21 days of Pi stress in roots. At present, the models account for elevation of IPS1 in response to $\mathrm{Pi}$ deficiency by having an extremely high rate of maximal synthesis $\left(m_{8}=696\right)$ and a larger Hill co-efficient $(r=4)$ than is normally used to model transcriptional regulation; ${ }^{26}$ though the latter could represent an amplification mechanism. However, both models poorly predict the repletion dynamics IPS1 (Fig. 5c), showing that something is not correct. Potentially, the high IPS1 levels could arise from either a Pi-sensitive "super-transcriptional complex" causing very high rates of synthesis or a Pi-sensitive protector that impedes IPS1 degradation. These shall be considered in turn.
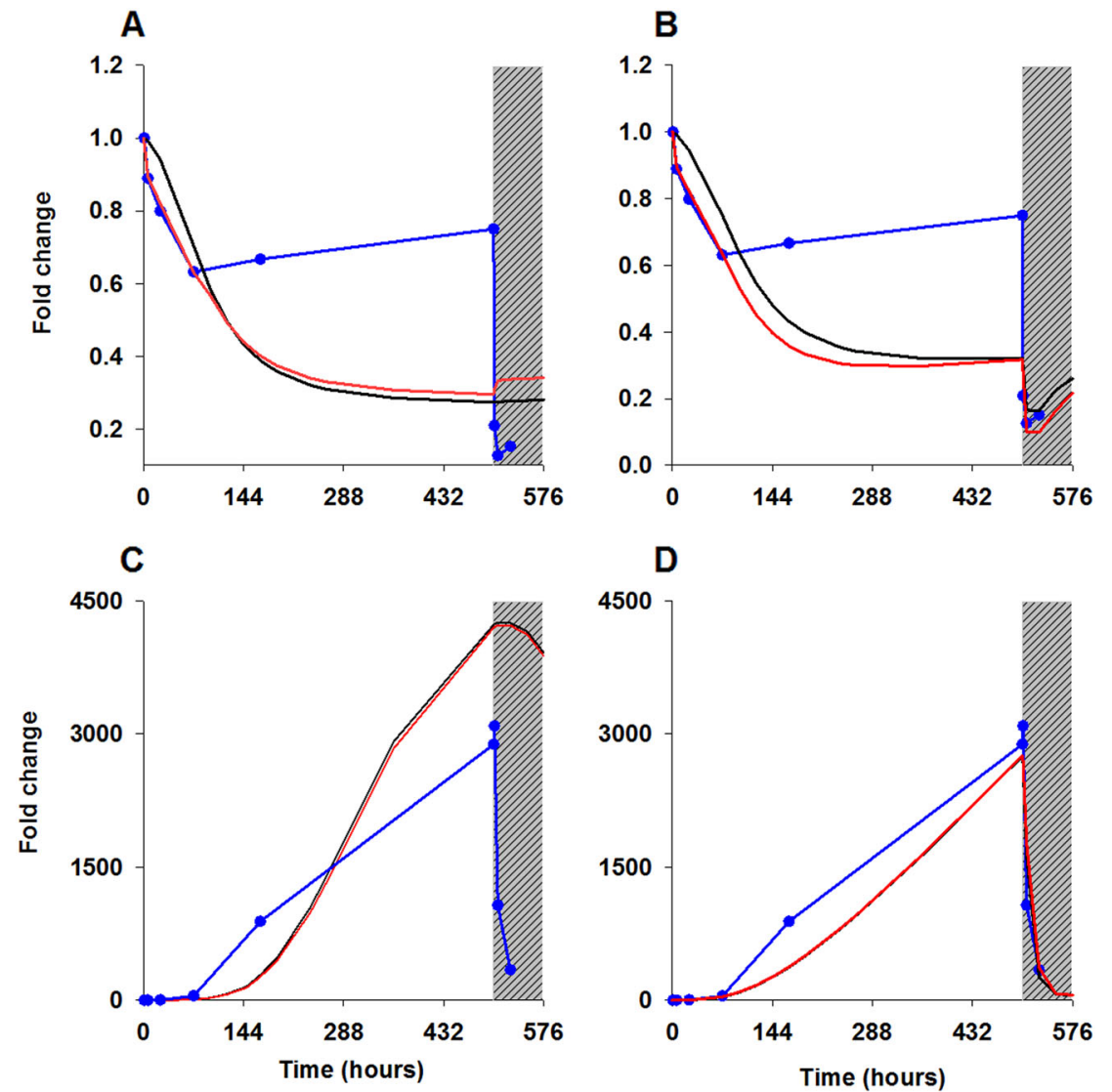

Fig. 5 Observed and predicted profiles of $\mathrm{PHO} 2$ and IPS1 transcripts under Pi-depletion and repletion conditions. Panels $\mathbf{a}$ and $\mathbf{b}$ correspond to PHO2, and $\mathbf{c}$ and $\mathbf{d}$ to IPS1. Panels $\mathbf{a}$ and $\mathbf{c}$ show predicted profiles of PiOM and PsTR models, while panels $\mathbf{b}$ and $\mathbf{d}$ depict models incorporating RNA-protection. The blue dashed lines show the fold change in RNA-SEQ values from. ${ }^{20}$ The red and black lines denote PsTR and PiOM models, respectively. The gray zones denote the period of Pi-resupply 
Poor evidence for a super-promoter upstream of IPS1

Recent work on auxin-response elements ${ }^{27}$ show how multiple transcription factor binding sites appear to act cooperatively rather than additively to cause higher levels of induction, with seven binding sites causing the maximum stimulation of almost 30 fold over constitutive expression levels. In the case of IPS1, transcription is $\sim 1000$ times higher than constitutive levels. Around the IPS1 promoter (see Supplementary Fig. 6), there are three copies of the P1BS motif-the binding sites for PHR2S. ${ }^{28}$ One of the P1BS sites is in the transcribed region, where RNA polymerase would at least temporarily separate the DNA strands, resulting in loss of PHR2S binding at that position. Furthermore, BLASTN searches with this upstream sequence revealed the presence of an unannotated tRNA-gly gene on the anti-sense strand. This makes the potential regulatory region of IPS1 shorter $(\sim 1200 \mathrm{bp})$, and data from ${ }^{20}$ indicate that the tRNA gene is also expressed in response to $\mathrm{Pi}$ stress, see Supplementary Fig. 7. Hence, this sequence analysis does not rule out the possibility of an initiator complex that allows extremely high rates of synthesis but it seems most unlikely.

Evidence for the protection of IPS1 RNA in Pi-deficient conditions RNA protection has not been reported in plants but there are two lines of evidence to support this hypothesis in the case of IPS1. First, RNA stability is known to be altered by protein binding. IPS1 is predicted to have two Pumilio binding sites near its 3 '-end, see Supplementary Fig. 6. The role of Pumilio-RNA-binding proteins is well understood in animal systems. ${ }^{29,30}$ Published transcriptomic data $^{20}$ show that the genes in this family are expressed at substantial levels and some may be elevated by Pi stress, see Supplementary Fig. 8. Another predicted protein-binding site near 3 '-end is for ELAV-like protein 1 (HuR). The nearest similar genes in plants are annotated as RNA-recognition and Poly-adenylate binding proteins, making these candidate IPS1-protectors as well.

The second line of evidence is from RNA structure prediction. Querying 3'-end subsequence of IPS1 RNA with different sizes of the poly-A tract in BLASTN searches against NCBI-Sequence Read Archive (SRX336041) showed that the IPS1 polyA tail was up to $\sim 18$ bases (see Supplementary Fig. 13). Predicted structures of IPS1, with and without an 18-base polyA tail are shown in Supplementary Fig. 9. These indicate that the poly-A tail considerably stabilises its structure through greatly increasing double-stranded (ds) RNA formation, which would make it more resistant to attack by ribonucleases. One can envisage a protein that binds to the polyA tail (or to the polyA-polyU dsRNA region) in the absence of $\mathrm{Pi}$. Upon repletion, this protein would no longer bind and IPS1 would revert to its original sensitivity to RNase degradation.

The 3'-end of IPS1 also has several stretches of complementary sequences, which could potentially form a pseudoknot, see Supplementary Fig. 10. Given that an 11 base-paired RNA sequence forms a complete cycle of a double helix, the 3 '-end of IPS1 can form a stem-loop consisting of two hydrogen bonding regions -6 and 11 base pairs long. The terminal loop contains AATAAAG that could form the pseudoknot, which could be stablised by the polyA tail hydrogen bonding to the $5^{\prime}$ sequence to form a 12 and, potentially, 4 base-paired polyA-polyT duplexes. Much smaller pseudo-knots are known to inhibit degradation by 3'exonucleases in plant RNA viruses. ${ }^{31,32}$ Potentially, it could play a role here. Considering everything, RNA protection is a more credible hypothesis and is worth investigating further.

\section{Models incorporating IPS1 RP}

Using the PiOM and PsTR models, the IPS1 protection hypothesis was tested by altering the degradation rates of IPS1 and IMC as functions of CytoPi and examining $\mathrm{Pi}$ repletion conditions after a period of Pi stress. Thus, the magnitude of the IPS1 degradation rate $\left(d_{7}^{\prime}\right)$ in the RP version of the models is altered and defined as the ratio of the original degradation rate of IPS1 $\left(d_{7}\right)$ and the steady-state initial CytoPi concentration under typical Pi condition (CytoPi, at time zero), as described in Supplementary Methods (Eqs 18-21). In these RP models, the degradation rates of IPS1 and IMC decreases in response to low $\mathrm{Pi}$, allowing IPS1 to accumulate and its reversible interaction with miR399 to form more IMC complexes. However, the degradation rates of IPS1 and IMC rapidly return to normal upon Pi re-supply. The sudden degradation of IMC will thereby cause a rapid increase in the pool of miR399 and a consequent short-term decline in the PHO2 mRNA levels. PiOM-RP and PsTR-RP model were fitted to the Pi-stress data set, reestimating the model parameters, see Supplementary Table 6.

Models substantiate the idea of IPS1 RP in Pi-deficient conditions Both PiOM-RP and PsTR-RP models fit the observed profiles of IPS1 and $\mathrm{PHO} 2$ under Pi depletion and repletion condition (Fig. $5 \mathrm{~d}, \mathrm{~b}$ ). While manually exploring the parameter space for the binding constant for IPS1-miR399 $\left(\mathrm{k}_{7}\right)$, the value in the range of $10^{-5} \mathrm{~s}^{-1}$ gave the best fit and thus was adjusted accordingly to $1.9 \mathrm{e}-05$, which is well still within the range of weak binding constants (i.e., $10^{-5} \mathrm{~s}^{-1}$ ) for an RNA-microRNA interaction. ${ }^{33}$ Notably, the reestimated parameters for IPS1 synthesis are significantly lower, particularly the Hill coefficient " $r$ " dropping to 2, but are in keeping with values often seen in models of gene regulation. ${ }^{25,26}$ These results endorse the idea of IPS1 RP under low Pi conditions and thus laboratory validation is the obvious next step. PsTR-RP is available from the BioModels database. ${ }^{34}$ Sensitivity and robustness analysis of all the models, with and without RP, were performed, as described in Supplementary Methods, and emphasize the very low robustness of IPS1, which presumably explains why its variable was not particularly sensitive to its Hill coefficient " $r$ ".

\section{DISCUSSION AND CONCLUSION}

This work has investigated the interface between local and systemic signals in rice roots experiencing phosphate starvation, in particular the mechanisms underlying the induced early loss of PHO2 mRNA, and the extreme elevation and steep decline of IPS1 RNA, upon Pi starvation and re-supply, respectively. Reproducing the known temporal dynamics of PHO2 and IPS1 transcripts, qRTPCR show delay in the appearance of miR399 compared to the early $\mathrm{PHO} 2$ mRNA loss in response to Pi starvation. This suggests the presence of an extra Pi-stress mechanism, causing an early loss in PHO2 mRNA levels.

A new mathematical model for the regulation of phosphate uptake in plants has been developed to test plausible hypotheses, concerning the regulation of $\mathrm{PHO} 2$ and IPS1 transcripts under different $\mathrm{Pi}$ conditions. Owing to the sparsity of the data, a nonlinear mixed-effect modeling approach ${ }^{35}$ was employed for parameter estimation of the original model (PiOM). The model correctly predicts the observed temporal dynamics of miR399 and IPS1 under Pi-sufficient and deficient condition. But, the prediction for PHO2 mRNA dynamics was poor with a 24-h delay in the early drop upon $\mathrm{Pi}$ starvation. This can be attributed to the slow appearance of miR399, which is known and modeled as the only degrader of $\mathrm{PHO} 2$ mRNA. To investigate the early loss of $\mathrm{PHO} 2$ mRNA under Pi stress, five hypothetical mechanisms were modeled. Among these, a PsTR is the most credible hypothesis on the basis of the improved overall fit to the data, fit to the observed 6-h time point value and the prior general understanding of allosteric regulation.

To aid resolution of the hypothetical mechanisms, qRT-PCR data were generated for four time points over initial $12 \mathrm{~h}$ following $\mathrm{Pi}$ starvation. Around $80 \%$ of $\mathrm{PHO} 2 \mathrm{mRNA}$ is lost by $3 \mathrm{~h}$, at a rate close to the normal degradation rate for RNA, and is partially 
restored by $6 \mathrm{~h}$ upon $\mathrm{Pi}$ stress. This is indicative of $\mathrm{PHO} 2$ regulating its own level of transcription, as the plant protein DELLA is known to regulate its own expression in a similar time scale. ${ }^{25}$ This leads to a hypothesis for regulation of $\mathrm{PHO} 2$ expression as shown in Supplementary Fig. 11.

Sequence analysis of the upstream region $500 \mathrm{bp}$ of $\mathrm{OsPHO} 2$ gene predicts various potential transcription factor binding sites. This largely includes signatures for MYB, WRKY, bHLH, bZIP, MADbox and DOF transcription factor families (see Supplementary Table 7). The expression profiles of the members from the corresponding transcription factor family under deficient $\mathrm{Pi}$ conditions are presented in Supplementary Fig. 12. The members in all the predicted transcription factor families show both similar and contrasting expression patterns to that of PHO2 mRNA in response to $\mathrm{Pi}$ starvation. However, experimental analyses are required to identity the $\mathrm{TA}$ of $\mathrm{PHO} 2$ and subsequent, characterization of its transcriptional inhibitor (TI).

In response to $\mathrm{Pi}$ re-supply, both $\mathrm{PiOM}$ and PsTR models incorrectly predict the observed steep drop in the levels of $\mathrm{PHO} 2$ and IPS1 RNAs, thus pointing to yet some other regulatory interaction not represented in these models. IPS1 RNA is known to sequester miR399 and accumulates to extremely high levels in plant roots during phosphate stress. Potentially, the sudden loss of IPS1 in response to Pi re-supply would release the bound miR399 causing the observed rapid loss of $\mathrm{PHO} 2$ mRNA. The observed IPS1 profiles can be explained by either the gene having a Pihypersensitive "super-promoter" synthesizing extremely high IPS1 RNA under low $\mathrm{Pi}$ conditions or the RNA being protected from degradation, ideally by Pi-sensitive RNA-binding proteins. Revised models incorporating RP favor the latter. Informatics analyses point to PUMILIO proteins and/or RNA secondary structure playing the role in IPS1 protection. However, exhaustive experimental investigation is required to explore this aspect of IPS1 dynamics. If confirmed, this would be the first case of regulation by RP in plant systems.

It is obviously desirable to extend systems approaches to other aspects of the phosphate-starvation response, including metabolic reprogramming. Evidence from Arabidopsis indicates that its PHR2 homolog is the key metabolic regulator, ${ }^{36,37}$ so the same is likely to be the case for rice. Furthermore, in rice PHR2 is known to promote the synthesis and secretion of enzymes and organic acids that promote mobilization of internal and external $\mathrm{Pi}^{38,39}$ Although it would be comparatively easy to extend the model to include PHR2 regulation of metabolism and re-cycling, it extremely difficult to estimate the corresponding model parameters. At present, the only available integrated transcriptomic and metabolomics rice study considers only one time point, ${ }^{40}$ and hence is of little value for parameter estimation. Indeed, this aspect of plant phosphate regulation deserves more research attention.

Sensitivity analysis shows that cytosolic Pi levels are most sensitive to the internal utilization rate $(U)$ and hence is another important aspect to study. However, this parameter covers a range of phenomena that require careful consideration, including vacuolar uptake, and incorporation into lipids and nucleic acids. The above metabolic reprogramming might be expected to reduce this utilization rate, and some vacuolar phosphate may be released back into cytosol. Furthermore, the above-mentioned exudates normally result in some extra Pi becoming available, allowing a higher rate of utilization. Inter-compartmental $\mathrm{Pi}$ dynamics would be fascinating to model but almost impossible to study in vivo, especially in intact plants. However, root mass continues to increase during Pi starvation. ${ }^{9}$ For all these reasons, this first modeling study has maintained a fixed utilization rate in order to gain a better understanding of the interactions in the local and systemic components of the starvation response.

In summary, this work has led to three hypotheses concerning the regulation of $\mathrm{Pi}$ uptake in rice: (1) $\mathrm{PHO} 2$ protein mediates the degradation of its own TA to maintain constant PHO2 mRNA levels; (2) The binding affinity of the TA of $\mathrm{PHO} 2$ is impaired by a phosphate-sensitive transcriptional repressor/inhibitor (TI); and (3) IPS1 RNA is protected from degradation by phosphate-sensitive RNA binding proteins. It is anticipated that this work will enable further studies and development in plant phosphate research, ultimately informing the development of crop varieties with improved Phosphorus Use Efficiencies.

\section{MATERIALS AND METHODS}

Plant materials and growth conditions

Rice (Oryza sativa cv. Nipponbare) was used across all the experiments. Seeds were first pre-germinated in a wet paper towel in tap water at $28^{\circ} \mathrm{C}$ for 5 days before being transferred into +Pi solution. Seeds For both the initial and later experiments, the 15-days old rice seedlings were transferred into the $-\mathrm{Pi}$ solution for 11 days and $24 \mathrm{~h}$, respectively. Hydroponic experiments were performed under controlled conditions (day/night temperature of $26 / 22^{\circ} \mathrm{C}$ and a $12-\mathrm{h}$ photoperiod, $200 \mu \mathrm{mol}$ photons $\mathrm{m}^{-2} \mathrm{~s}^{-1}$ ) using a randomized experimental design, allowing $0.5 \mathrm{~L}$ of hydroponic solution per plant. The rice culture solution previously described $\mathrm{in}^{20}$ was used in the hydroponic growing system. For Pisufficient (+Pi) condition, $320 \mu \mathrm{M} \mathrm{NaH}{ }_{2} \mathrm{PO}_{4}$ was used. While, $\mathrm{NaH}_{2} \mathrm{PO}_{4}$ was replaced by $320 \mu \mathrm{M} \mathrm{NaCl}$ for Pi-deficient $(-\mathrm{Pi})$ solution. The $\mathrm{pH}$ of the solution was adjusted to 5.5, and renewed every 3 days. All experiment procedures such as media replacement and sample collection were performed at a similar time of the day to minimize possible circadian effects.

\section{RNA isolation and qRT-PCR analysis}

Total RNA from frozen root samples, pooled from five plants, was isolated using TRIzol reagent (Life Technologies) followed by treatment with DNase I (Qiagen) and column clean-up using the RNeasy mini kit (QIAGEN) to eliminate genomic DNA contamination. CDNA was synthesized using $5 \mu \mathrm{L}$ of total RNA (500 ng) using SuperScript III First-Strand Synthesis kit (Invitrogen, Catalog No. 18080-400). The synthesized CDNA is cleaned using an enzyme mix included in the kit (E. coli RNase H). qRT-PCR was performed using PerfeCTa qPCR SuperMix on a LightCycler 480 Real-Time PCR system (Roche) according to the manufacturer's instructions. Relative expression levels were normalized to that of an internal control, Os-ACTIN. qRT-PCR for quantification of mature miR399 was performed following a published protocol ${ }^{41}$ and the corresponding primers are published in. ${ }^{10}$ PHO2, IPS1 and Actin primers used for qRT-PCR are listed in Supplementary Table 8. For Fig. 1 , the experiments conducted with $n$ (biological replicates) $=2$ for IPS1 and miR399 at 0,24 , and $72 \mathrm{~h} ; n=4$ for IPS1 and miR399 at 168 and $264 \mathrm{~h}$, and $n=3$ for PHO2. For all other qRT-PCR experiments three biological replicates were used.

\section{Parameter estimation}

The unknown model parameters were inferred by non-linear mixed-effects models implemented in the software named MONOLIX (MOdèles NOnLInéaires à effects miXtes), version $4.33 \mathrm{~s}^{35}$ freely available at (http:// www.lixoft.eu/). This software consists of algorithms that combine the stochastic approximation of expectation maximization algorithm with a Markov chain Monte Carlo procedure to estimate the maximum likelihood of the model parameters without any linearisation techniques. In MONOLIX, the statistical models are evaluated by using analytical model selection tools, which includes information criteria such as AIC and Bayesian information criterion, and statistical tests such as Likelihood Ratio test and Wald Test. Such evaluation tools allow the building of improved statistical models, enhancing the precision of the estimates for the parameters. More details on model fitting using MONOLIX are given in Supplementary Methods.

\section{Centroid secondary structure prediction of IPS1 mRNA}

RNA structures were generated using RNAfold (rna.tbi.univie.ac.at) with default settings. Centroid, rather than minimum free-energy, structures were generated as these show the most probable base-pairing regions in the ensemble of secondary structures likely to be found near the most thermodynamically stable conformation. 
Data availability

The data and figures generated during this study are available in FigShare (https://figshare.com/projects/Regulatory_feedback_response_mechanism s_to_phosphate_starvation_in_rice/26098). The mathematical model is available at Biomodels with ID MODEL1702210000 (http://www.ebi.ac.uk/ biomodels/).

\section{ACKNOWLEDGEMENTS}

We acknowledge input from Mudassar lqbal, Jean-Louis Dinh and Mohammed Cherkaoui Rbati (on parameter estimation), and Faraz Khan and Lu Jiang (on laboratory procedures). This work was supported by the Biotechnology and Biological Sciences Research Council [grant number BB/J020443/1], a Vice-Chancellor Research Excellence Award (\#598) and Building Experience/Skills Travel (BEST) Award from University of Nottingham (UK) and Gen Foundation scholarship (to IA), and a University of Nottingham/Zhejiang University collaborative project grant (to TCH and $\mathrm{CL})$.

\section{AUTHOR CONTRIBUTIONS}

I.A., C.L., P.W. and T.C.H. designed research; I.A., J.S. and J.G. performed laboratory experiments; I.A. carried out the mathematical modeling; I.A., D.J.S., C.L. and T.C.H. wrote the paper; all authors approved the final version. The funders had no role in study design, data collection and analysis, decision to publish, or preparation of the manuscript.

\section{ADDITIONAL INFORMATION}

Supplementary information accompanies the paper on the npj Systems Biology and Applications website (https://doi.org/10.1038/s41540-017-0041-0).

Competing interests: The authors declare no competing financial interests. Publisher's note: Springer Nature remains neutral with regard to jurisdictional claims in published maps and institutional affiliations.

\section{REFERENCES}

1. Schachtman, D. P., Reid, R. J. \& Ayling, S. M. Phosphorus uptake by plants: from soil to cell. Plant Physiol. 116, 447-453 (1998).

2. Rouached, H., Arpat, A. B. \& Poirier, Y. Regulation of phosphate starvation responses in plants: signaling players \& cross-talks. Mol. Plant 3, 288-299 (2010).

3. Niu, Y. F. et al. Responses of root architecture development to low phosphorus availability: a review. Ann. Bot. 112, 391-408 (2012).

4. Gilbert, N. Environment: the disappearing nutrient. Nature 461, 716-718 (2009).

5. Elser, J. J. Phosphorus: a limiting nutrient for humanity? Curr. Opin. Biotechnol. 23, 833-838 (2012).

6. Veneklaas, E. J. et al. Opportunities for improving phosphorus-use efficiency in crop plants. New Phytol. 195, 306-320 (2012).

7. Wu, P. et al. Improvement of phosphorus efficiency in rice on the basis of underst\&ing phosphate signaling \& homeostasis. Curr. Opin. Plant Biol. 16, 205-212 (2013).

8. Lopez-Arredondo, D. L. et al. Phosphate nutrition: improving low-phosphate tolerance in crops. Annu. Rev. Plant Biol. 65, 95-123 (2014).

9. Lambers, H. \& Plaxton,W. C. in Annual Plant Reviews, Vol. 48 (eds Plaxton, W. C. \& Lambers, H.) Ch. 1 (Wiley, Chichester, UK, 2015).

10. Lv, Q. et al. SPX4 negatively regulates phosphate signaling \& homeostasis through its interaction with PHR2 in rice. Plant Cell. 26, 1586-1597 (2014).

11. Miura, K. et al. The Arabidopsis SUMO E3 ligase SIZ1 controls phosphate deficiency responses. Proc. Natl. Acad. Sci. USA 102, 7760-7765 (2005).

12. Aung, K. et al. pho2, a phosphate overaccumulator, is caused by a nonsense mutation in a microRNA399 target gene. Plant Physiol. 141, 1000-1011 (2006).

13. Bari, R. et al. PHO2, microRNA399, \& PHR1 define a phosphate-signaling pathway in plants. Plant Physiol. 141, 988-999 (2006).

14. Hu, B. et al. LEAF TIP NECROSIS1 plays a pivotal role in the regulation of multiple phosphate starvation responses in rice. Plant Physiol. 156, 1101-1115 (2011).

15. Liu, T. Y. et al. PHO2-dependent degradation of PHO1 modulates phosphate homeostasis in Arabidopsis. Plant Cell. 24, 2168-2183 (2012).

16. Arpat, A. B. et al. Functional expression of PHO1 to the Golgi \& trans-Golgi network \& its role in export of inorganic phosphate. Plant J. 71, 479-491 (2012).

17. Hou, X. L. et al. Regulation of the expression of OsIPS1 \& OsIPS2 in rice via systemic \& local Pi signalling \& hormones. Plant Cell. Environ. 28, 353-364 (2005).

18. Shin, $\mathrm{H}$. et al. Loss of At4 function impacts phosphate distribution between the roots \& the shoots during phosphate starvation. Plant J. 45, 712-726 (2006).
19. Franco-Zorrilla, J. M. et al. Target mimicry provides a new mechanism for regulation of microRNA activity. Nat. Genet. 39, 1033-1037 (2007).

20. Secco, D. et al. Spatio-temporal transcript profiling of rice roots \& shoots in response to phosphate starvation \& recovery. Plant. Cell. 25, 4285-4304 (2013).

21. Oono, Y. et al. mRNA-Seq reveals a comprehensive transcriptome profile of rice under phosphate stress. Rice 4, 50-65 (2011).

22. Miura, K. et al. SIZ1 regulation of phosphate starvation-induced root architecture remodeling involves the control of auxin accumulation. Plant Physiol. 155, 1000-1012 (2011)

23. Chiou, T. J. The role of microRNAs in sensing nutrient stress. Plant Cell. Environ. 30, 323-332 (2007)

24. Kuo, H. F. \& Chiou, T. J. The role of microRNAs in phosphorus deficiency signaling. Plant Physiol. 156, 1016-1024 (2011).

25. Band, L. R. et al. Growth-induced hormone dilution can explain the dynamics of plant root cell elongation. Proc. Natl. Acad. Sci. USA 109, 7577-7582 (2012).

26. Muraro, D. et al. Integration of hormonal signaling networks \& mobile microRNAs is required for vascular patterning in Arabidopsis roots. Proc. Natl. Acad. Sci. USA 111, 857-862 (2014).

27. Mironova, V. V. et al. How multiple auxin responsive elements may interact in plant promoters: a reverse problem solution. J. Bioinform. Comput. Biol. 11, 1340011 (2013).

28. Rubio, V. et al. A conserved MYB transcription factor involved in phosphate starvation signaling both in vascular plants \& in unicellular algae. Genes. Dev. 15, 2122-2133 (2001).

29. Spassov, D. S. \& Jurecic, R. The PUF family of RNA-binding proteins: does evolutionarily conserved structure equal conserved function? IUBMB Life 55, 359-366 (2003).

30. Zamore, P. D., Williamson, J. R. \& Lehmann, R. The Pumilio protein binds RNA through a conserved domain that defines a new class of RNA-binding proteins. RNA 3, 1421-1433 (1997).

31. Gallie, D. R. \& Walbot, V. RNA pseudoknot domain of tobacco mosaic virus can functionally substitute for a poly(A) tail in plant \& animal cells. Genes. Dev. 4, 1149-1157 (1990)

32. van Belkum, A. et al. Five pseudoknots are present at the 204 nucleotides long $3^{\prime}$ noncoding region of tobacco mosaic virusRNA. Nucleic Acids Res. 13, 7673-7686 (1985).

33. Zinovyev, A. et al. Mathematical modeling of microRNA-mediated mechanisms of translation repression. Adv. Exp. Med. Biol. 774, 189-224 (2013).

34. Chelliah, V. et al. BioModels: ten-year anniversary. Nucl. Acids Res. 43, D542-D548 (2015).

35. Laveille, M. Mixed Effects Models for the Population Approach: Models, Tasks, Methods and Tools (Chapman and Hall/CRC, Boca Raton, FL, 2014).

36. Pant, B. D. et al. Identification of primary \& secondary metabolites with phosphorus status-dependent abundance in Arabidopsis, \& of the transcription factor PHR1 as a major regulator of metabolic changes during phosphorus limitation. Plant Cell. Environ. 38, 172-187 (2015).

37. Pant, B. D. et al. The transcription factor PHR1 regulates lipid remodeling \& triacylglycerol accumulation in Arabidopsis thaliana during phosphorus starvation. J. Exp. Bot. 66, 1907-1918 (2015).

38. Pandey, B. K. et al. OsHAD1, a haloacid dehalogenase-like APase enhances phosphate accumulation. Plant Physiol. 174, 2316-2332 (2017).

39. Mehra, P. et al. Improvement in phosphate acquisition \& utilization by a secretory purple acid phosphatase (OsPAP21b). in rice. Plant. Biotechnol. J. 15, 1054-1067 (2017).

40. Mehra, P. et al. Comparative morphophysiological analyses \& molecular profiling reveal Pi-efficient strategies of a traditional rice genotype. Front. Plant Sci. 8, 1184 (2016).

41. Varkonyi-Gasic, E. et al. Protocol: a highly sensitive RT-PCR method for detection \& quantification of microRNAs. Plant. Methods 3, 12 (2007).

42. Murtaugh, P. A. In defense of P values. Ecology 95, 611-617 (2014).

Open Access This article is licensed under a Creative Commons Attribution 4.0 International License, which permits use, sharing, adaptation, distribution and reproduction in any medium or format, as long as you give appropriate credit to the original author(s) and the source, provide a link to the Creative Commons license, and indicate if changes were made. The images or other third party material in this article are included in the article's Creative Commons license, unless indicated otherwise in a credit line to the material. If material is not included in the article's Creative Commons license and your intended use is not permitted by statutory regulation or exceeds the permitted use, you will need to obtain permission directly from the copyright holder. To view a copy of this license, visit http://creativecommons. org/licenses/by/4.0/.

(c) The Author(s) 2017 